\title{
Fast-Robust PCA
}

\author{
Markus Storer, Peter M. Roth, Martin Urschler, and Horst Bischof \\ Institute for Computer Graphics and Vision, \\ Graz University of Technology, \\ Inffeldgasse 16/II, 8010 Graz, Austria \\ \{storer, pmroth, urschler, bischof\}@icg.tugraz . at
}

\begin{abstract}
Principal Component Analysis (PCA) is a powerful and widely used tool in Computer Vision and is applied, e.g., for dimensionality reduction. But as a drawback, it is not robust to outliers. Hence, if the input data is corrupted, an arbitrarily wrong representation is obtained. To overcome this problem, various methods have been proposed to robustly estimate the PCA coefficients, but these methods are computationally too expensive for practical applications. Thus, in this paper we propose a novel fast and robust PCA (FR-PCA), which drastically reduces the computational effort. Moreover, more accurate representations are obtained. In particular, we propose a two-stage outlier detection procedure, where in the first stage outliers are detected by analyzing a large number of smaller subspaces. In the second stage, remaining outliers are detected by a robust least-square fitting. To show these benefits, in the experiments we evaluate the FR-PCA method for the task of robust image reconstruction on the publicly available ALOI database. The results clearly show that our approach outperforms existing methods in terms of accuracy and speed when processing corrupted data.
\end{abstract}

\section{Introduction}

Principal Component Analysis (PCA) 1] also known as Karhunen-Loève transformation (KLT) is a well known and widely used technique in statistics. The main idea is to reduce the dimensionality of data while retaining as much information as possible. This is assured by a projection that maximizes the variance but minimizes the mean squared reconstruction error at the same time. Murase and Nayar 2 showed that high dimensional image data can be projected onto a subspace such that the data lies on a lower dimensional manifold. Thus, starting from face recognition (e.g., 34]) PCA has become quite popular in computer vision 1, where the main application of PCA is dimensionality reduction. For instance, a number of powerful model-based segmentation algorithms such as Active Shape Models [8] or Active Appearance Models [9] incorporate PCA as a fundamental building block.

In general, when analyzing real-world image data, one is confronted with unreliable data, which leads to the need for robust methods (e.g., [10]1]). Due to

${ }^{1}$ For instance, at CVPR 2007 approximative $30 \%$ of all papers used PCA at some point (e.g., [5617]). 
its least squares formulation, PCA is highly sensitive to outliers. Thus, several methods for robustly learning PCA subspaces (e.g., [12 13141516]) as well as for robustly estimating the PCA coefficients (e.g., 17,18 19 20]) have been proposed. In this paper, we are focusing on the latter case. Thus, in the learning stage a reliable model is estimated from undisturbed data, which is then applied to robustly reconstruct unreliable values from the unseen corrupted data.

To robustly estimate the PCA coefficients Black and Jepson [18] applied an Mestimator technique. In particular, they replaced the quadratic error norm with a robust one. Similarly, Rao [17] introduced a new robust objective function based on the MDL principle. But as a disadvantage, an iterative scheme (i.e., EM algorithm) has to be applied to estimate the coefficients. In contrast, Leonardis and Bischof 19 proposed an approach that is based on sub-sampling. In this way, outlying values are discarded iteratively and the coefficients are estimated from inliers only. Similarly, Edwards and Murase introduced adaptive masks to eliminate corrupted values when computing the sum-squared errors.

A drawback of these methods is their computational complexity (i.e., iterative algorithms, multiple hypotheses, etc.), which limits their practical applicability. Thus, we develop a more efficient robust PCA method that overcomes this limitation. In particular, we propose a two-stage outlier detection procedure. In the first stage, we estimate a large number of smaller subspaces sub-sampled from the whole dataset and discard those values that are not consistent with the subspace models. In the second stage, the data vector is robustly reconstructed from the thus obtained subset. Since the subspaces estimated in the first step are quite small and only a few iterations of the computationally more complex second step are required (i.e., most outliers are already discarded by the first step), the whole method is computationally very efficient. This is confirmed by the experiments, where we show that the proposed method outperforms existing methods in terms of speed and accuracy.

This paper is structured as follows. In Section 2, we introduce and discuss the novel fast-robust PCA (FR-PCA) approach. Experimental results for the publicly available ALOI database are given in Section 3 . Finally, we discuss our findings and conclude our work in Section 4

\section{Fast-Robust PCA}

Given a set of $n$ high-dimensional data points $\mathbf{x}_{j} \in \mathbb{R}^{m}$ organized in a matrix $\mathbf{X}=\left[\mathbf{x}_{1}, \ldots, \mathbf{x}_{n}\right] \in \mathbb{R}^{m \times n}$, then the PCA basis vectors $\mathbf{u}_{1}, \ldots, \mathbf{u}_{n-1}$ correspond to the eigenvectors of the sample covariance matrix

$$
\mathbf{C}=\frac{1}{n-1} \hat{\mathbf{X}} \hat{\mathbf{X}}^{\top}
$$

where $\hat{\mathbf{X}}=\left[\hat{\mathbf{x}}_{1}, \ldots, \hat{\mathbf{x}}_{n}\right]$ is the mean normalized data with $\hat{\mathbf{x}}_{j}=\mathbf{x}_{j}-\overline{\mathbf{x}}$. The sample mean $\overline{\mathbf{x}}$ is calculated by

$$
\overline{\mathbf{x}}=\frac{1}{n} \sum_{j=1}^{n} \mathbf{x}_{j}
$$


Given the PCA subspace $\mathbf{U}_{p}=\left[\mathbf{u}_{1}, \ldots, \mathbf{u}_{p}\right]$ (usually only $p, p<n$, eigenvectors are sufficient), an unknown sample $\mathbf{x} \in \mathbb{R}^{m}$ can be reconstructed by

$$
\tilde{\mathbf{x}}=\mathbf{U}_{p} \mathbf{a}+\overline{\mathbf{x}}=\sum_{j=1}^{p} a_{j} \mathbf{u}_{j}+\overline{\mathbf{x}}
$$

where $\tilde{\mathbf{x}}$ denotes the reconstruction and $\mathbf{a}=\left[a_{1}, \ldots, a_{p}\right]$ are the PCA coefficients obtained by projecting $\mathbf{x}$ onto the subspace $\mathbf{U}_{p}$.

If the sample $\mathbf{x}$ contains outliers, Eq. (3) does not yield a reliable reconstruction; a robust method is required (e.g., [171819 20]). But since these methods are computationally very expensive (i.e., they are based on iterative algorithms) or can handle only a small amount of noise, they are often not applicable in practice. Thus, in the following we propose a new fast robust PCA approach (FR-PCA), which overcomes these problems.

\subsection{FR-PCA Training}

The training procedure, which is sub-divided into two major parts, is illustrated in Figure 1. First, a standard PCA subspace $\mathbf{U}$ is generated using the full available training data. Second, $N$ sub-samplings $\mathbf{s}_{n}$ are established from randomly selected values from each data point (illustrated by the red points and the green crosses). For each sub-sampling $\mathbf{s}_{n}$, a smaller subspace (sub-subspace) $\mathbf{U}^{n}$ is estimated, in addition to the full subspace.

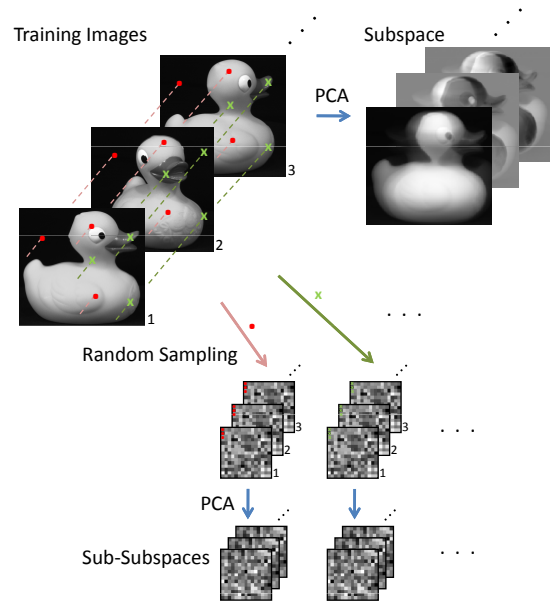

Fig. 1. FR-PCA training: A global PCA subspace and a large number of smaller PCA sub-subspaces are estimated in parallel. Sub-subspaces are derived by randomly subsampling the input data. 


\section{$2.2 \quad$ FR-PCA Reconstruction}

Given a new unseen test sample $\mathbf{x}$, the robust reconstruction $\tilde{\mathbf{x}}$ is estimated in two stages. In the first stage (gross outlier detection), the outliers are detected based on the reconstruction errors of the sub-subspaces. In the second stage (refinement), using the thus estimated inliers, a robust reconstruction $\tilde{\mathbf{x}}$ of the whole sample is generated.

In the gross outlier detection, first, $N$ sub-samplings $\mathbf{s}_{n}$ are generated according to the corresponding sub-subspaces $\mathbf{U}^{n}$, which were estimated as described in Section 2.1. In addition, we define the set of "inliers" $\mathbf{r}$ as the union of all selected pixels: $\mathbf{r}=\mathbf{s}_{1} \cup \ldots \cup \mathbf{s}_{N}$, which is illustrated in Figure 2(a) (green points). Next, for each sub-sampling $\mathbf{s}_{n}$ a reconstruction $\tilde{\mathbf{s}}_{n}$ is estimated by Eq. (3), which allows to estimate the error-maps

$$
\mathbf{e}_{n}=\left|\mathbf{s}_{n}-\tilde{\mathbf{s}}_{n}\right|,
$$

the mean reconstruction error $\bar{e}$ over all sub-samplings, and the mean reconstruction errors $\bar{e}_{n}$ for each of the $N$ sub-samplings.

Based on these errors, we can detect the outliers by local and global thresholding. The local thresholds (one for each sub-sampling) are defined by $\theta_{n}=\bar{e}_{n} w_{n}$, where $w_{n}$ is a weighting parameter and the global threshold $\theta$ is set to the mean error $\bar{e}$. Then, all points $s_{n,(i, j)}$ for which

$$
e_{n,(i, j)}>\theta_{n} \quad \text { or } \quad e_{n,(i, j)}>\theta
$$

are discarded from the sub-samplings $\mathbf{s}_{n}$ obtaining $\hat{\mathbf{s}}_{n}$. Finally, we re-define the set of "inliers" by

$$
\mathbf{r}=\hat{\mathbf{s}}_{1} \cup \ldots \cup \hat{\mathbf{s}}_{q},
$$

where $\hat{\mathbf{s}}_{1}, \ldots, \hat{\mathbf{s}}_{q}$ indicate the first $q$ sub-samplings (sorted by $\bar{e}_{n}$ ) such that $|\mathbf{r}| \leq$ $k ; k$ is the pre-defined maximum number of points. The thus obtained "inliers" are shown in Figure 2(b).

The gross outlier detection procedure allows to remove most outliers. Thus, the obtained set $\mathbf{r}$ contains almost only inliers. To further improve the final result in the refinement step, the final robust reconstruction is estimated similar to [19. Starting from the point set $\mathbf{r}=\left[r_{1}, \ldots, r_{k}\right], k>p$, obtained from the gross outlier detection, repeatedly reconstructions $\tilde{\mathbf{x}}$ are computed by solving an over-determined system of equations minimizing the least squares reconstruction error

$$
E(\mathbf{r})=\sum_{i=1}^{k}\left(x_{r_{i}}-\sum_{j=1}^{p} a_{j} \mathbf{u}_{j, r_{i}}\right)^{2} .
$$

Thus, in each iteration those points with the largest reconstruction errors can be discarded from $\mathbf{r}$ (selected by a reduction factor $\alpha$ ). These steps are iterated until a pre-defined number of remaining points is reached. Finally, an outlier-free subset is obtained, which is illustrated in Figure 2(c).

A robust reconstruction result obtained by the proposed approach compared to a non-robust method is shown in Figure 3 . One can clearly see that the robust 


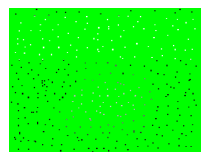

(a)

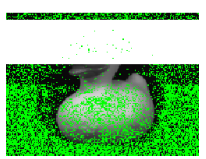

(b)

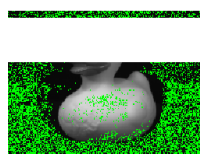

(c)

Fig. 2. Data point selection process: (a) data points sampled by all sub-subspaces, (b) occluded image showing the remaining data points after applying the sub-subspace procedure, and (c) resulting data points after the iterative refinement process for the calculation of the PCA coefficients. This figure is best viewed in color.

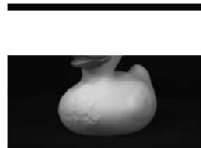

(a)

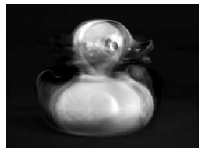

(b)

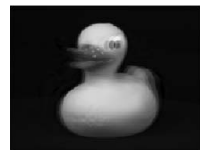

(c)

Fig. 3. Demonstration of the insensitivity of the robust PCA to noise (i.e., occlusions): (a) occluded image, (b) reconstruction using standard PCA, and (c) reconstruction using the FR-PCA

method considerably outperforms the standard PCA. Note, the blur visible in the reconstruction of the FR-PCA is the consequence of taking into account only a limited number of eigenvectors.

In general, the robust estimation of the coefficients is computationally very efficient. In the gross outlier detection procedure, only simple matrix operations have to be performed, which are very fast; even if hundreds of sub-subspace reconstructions have to be computed. The computationally more expensive part is the refinement step, where repeatedly an overdetermined linear system of equations has to be solved. Since only very few refinement iterations have to be performed due to the preceding gross outlier detection, the total runtime is kept low.

\section{Experimental Results}

To show the benefits of the proposed fast robust PCA method (FR-PCA), we compare it to the standard PCA (PCA) and the robust PCA approach presented in 19] (R-PCA). We choose the latter one, since it yields superior results among the presented methods in the literature and our refinement process is similar to theirs.

In particular, the experiments are evaluated for the task of robust image reconstruction on the "Amsterdam Library of Object Images (ALOI)" database [21. The ALOI database consists of 1000 different objects. Over hundred images of each object are recorded under different viewing angles, illumination angles and illumination colors, yielding a total of 110,250 images. For our experiments we arbitrarily choose 30 categories (009, 018, 024, 032, 043, 074, 090, 093, 125, 127, 

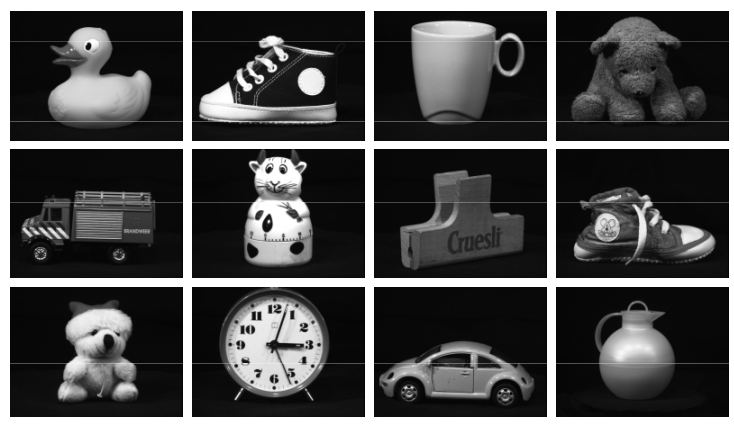

Fig. 4. Illustrative examples of ALOI database objects [21] used in the experiments

$135,138,151,156,171,174,181,200,299,306,323,354,368,376,409,442,602$, $809,911,926)$, where an illustrative subset of objects is shown in Figure 4.

In our experimental setup, each object is represented in a separate subspace and a set of 1000 sub-subspaces, where each sub-subspace contains $1 \%$ of data points of the whole image. The variance retained for the sub-subspaces is $95 \%$ and $98 \%$ for the whole subspace, which is also used for the standard PCA and the R-PCA. Unless otherwise noted, all experiments are performed with the parameter settings given in Table 1 .

Table 1. Parameters for the FR-PCA (a) and the R-PCA (b) used for the experiments

(a)

\begin{tabular}{|c|c|}
\hline \multicolumn{1}{|c|}{ FR-PCA } & \\
\hline Number of initial points $k$ & $130 p$ \\
\hline Reduction factor $\alpha$ & 0.9 \\
\hline
\end{tabular}

(b)

\begin{tabular}{|l|c|}
\hline \multicolumn{1}{|c|}{ R-PCA } & \\
\hline Number of initial hypotheses H & 30 \\
\hline Number of initial points k & $48 p$ \\
\hline Reduction factor $\alpha$ & 0.85 \\
\hline K2 & 0.01 \\
\hline Compatibility threshold & 100 \\
\hline
\end{tabular}

A 5-fold cross-validation is performed for each object category, resulting in $80 \%$ training- and $20 \%$ test data, corresponding to 21 test images per iteration. The experiments are accomplished for several levels of spatially coherent occlusions and several levels of salt \& pepper noise. Quantitative results for the root-mean-squared (RMS) reconstruction-error per pixel for several levels of occlusions are given in Table 2 In addition, in Figure 5 we show box-plots of the RMS reconstruction-error per pixel for different levels of occlusions. Analogously, the RMS reconstruction-error per pixel for several levels of salt \& pepper noise is presented in Table 3 and the corresponding box-plots are shown in Figure 6 .

From Table 2 and Figure 5 it can be seen - starting from an occlusion level of $0 \%$ - that all subspace methods exhibit nearly the same RMS reconstructionerror. Increasing the portion of occlusion, the standard PCA shows large errors 
Table 2. Comparison of the reconstruction errors of the standard PCA, the R-PCA and the FR-PCA for several levels of occlusion showing RMS reconstruction-error per pixel given by mean and standard deviation

\begin{tabular}{|c|c|c|c|c|c|c|c|c|c|c|c|c|}
\hline \multirow{3}{*}{ Occlusion } & \multicolumn{12}{|c|}{ Error per Pixel } \\
\hline & \multicolumn{2}{|c|}{$0 \%$} & \multicolumn{2}{|c|}{$10 \%$} & \multicolumn{2}{|c|}{$20 \%$} & \multicolumn{2}{|c|}{$30 \%$} & \multicolumn{2}{|c|}{$50 \%$} & \multicolumn{2}{|c|}{$70 \%$} \\
\hline & mean & std & mean & std & mean & std & mean & std & mean & std & mean & std \\
\hline PCA & 10.06 & 6.20 & 21.82 & 8.18 & 35.01 & 12.29 & 48.18 & 15.71 & 71.31 & 18.57 & 92.48 & 18.73 \\
\hline R-PCA & 11.47 & 7.29 & 11.52 & 7.31 & 12.43 & 9.24 & 22.32 & 21.63 & 59.20 & 32.51 & 94.75 & 43.13 \\
\hline FR-PCA & 10.93 & 6.61 & 11.66 & 6.92 & 11.71 & 6.95 & 11.83 & 7.21 & 26.03 & 23.05 & 83.80 & 79.86 \\
\hline
\end{tabular}

Table 3. Comparison of the reconstruction errors of the standard PCA, the R-PCA and the FR-PCA for several levels of salt \& pepper noise showing RMS reconstructionerror per pixel given by mean and standard deviation

\begin{tabular}{|c|c|c|c|c|c|c|c|c|c|c|}
\hline \multirow{3}{*}{ Salt\&Pepper Noise } & \multicolumn{10}{|c|}{ Error per Pixel } \\
\hline & \multicolumn{2}{|c|}{$10 \%$} & \multicolumn{2}{|c|}{$20 \%$} & \multicolumn{2}{|c|}{$30 \%$} & \multicolumn{2}{|c|}{$50 \%$} & \multicolumn{2}{|c|}{$70 \%$} \\
\hline & mean & std & mean & std & mean & std & mean & std & mean & std \\
\hline PCA & 11.77 & 5.36 & 14.80 & 4.79 & 18.58 & 4.80 & 27.04 & 5.82 & 36.08 & 7.48 \\
\hline R-PCA & 11.53 & 7.18 & 11.42 & 7.17 & 11.56 & 7.33 & 11.63 & 7.48 & 15.54 & 10.15 \\
\hline FR-PCA & 11.48 & 6.86 & 11.30 & 6.73 & 11.34 & 6.72 & 11.13 & 6.68 & 14.82 & 7.16 \\
\hline
\end{tabular}

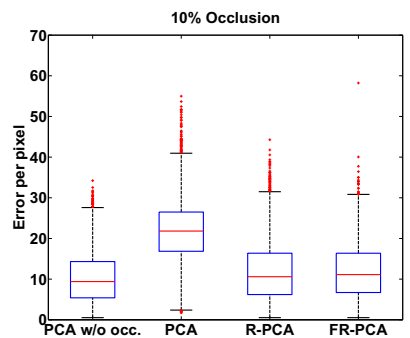

(a)

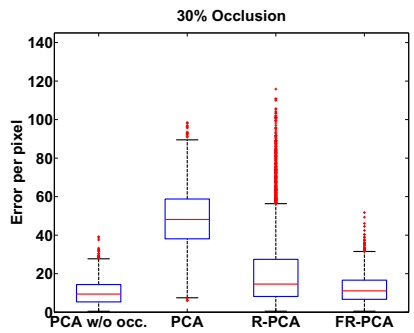

(c)

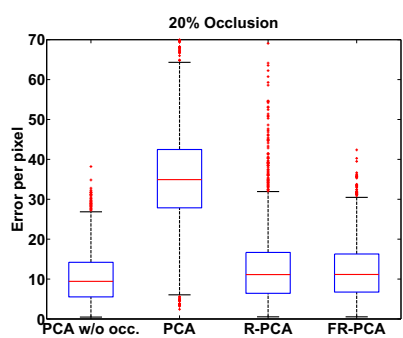

(b)

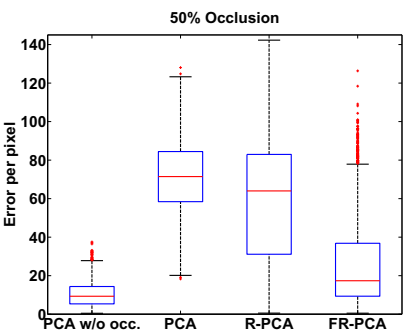

(d)

Fig. 5. Box-plots for different levels of occlusions for the RMS reconstruction-error per pixel. PCA without occlusion is shown in every plot for the comparison of the robust methods to the best feasible reconstruction result. 


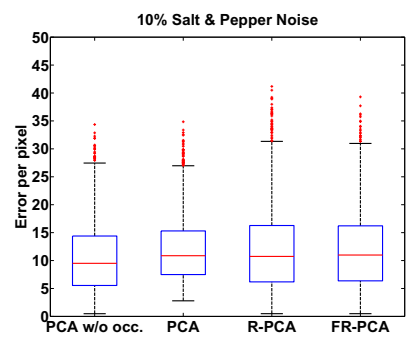

(a)

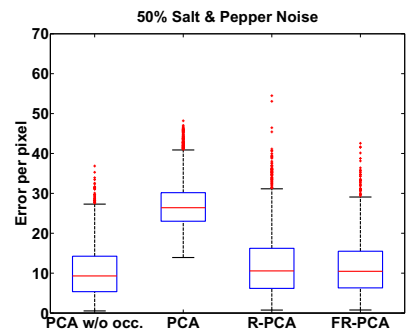

(c)

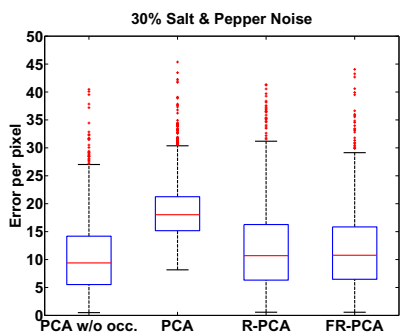

(b)

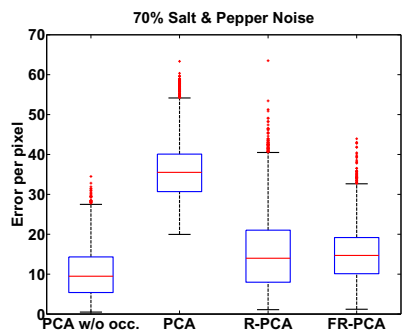

(d)

Fig. 6. Box-plots for different levels of salt \& pepper noise for the RMS reconstructionerror per pixel. PCA without occlusion is shown in every plot for the comparison of the robust methods to the best feasible reconstruction result.

whereas the robust methods are still comparable to the non-disturbed (best feasible) case, where our novel FR-PCA presents the best performance. In contrast, as can be seen from Table 3 and Figure [6, all methods can generally cope better with salt \& pepper noise. However, also for this experiment FR-PCA yields the best results.

Finally, we evaluated the runtime 1 for the applied different PCA reconstruc-

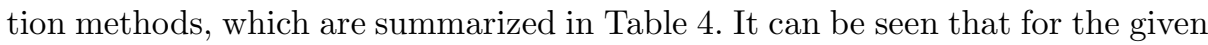
setup compared to R-PCA for a comparable reconstruction quality the robust reconstruction can be speeded up by factor of 18 ! This drastic speed-up can be explained by the fact that the refinement process is started from a set of data points mainly consisting of inliers. In contrast, in [19] several point sets (hypotheses) have to be created and the iterative procedure has to be run for every set resulting in a poor runtime performance. Reducing the number of hypotheses or the number of initial points would decrease the runtime, but, however, the reconstruction accuracy gets worse. In particular, the runtime of our approach only depends slightly on the number of starting points, thus having nearly constant execution times. Clearly, the runtime depends on the number and size of used eigenvectors. Increasing one of those values, the gap between the runtime for both methods is even getting larger.

${ }^{1}$ The runtime is measured in MATLAB using an Intel Xeon processor running at $3 \mathrm{GHz}$. The resolution of the images is $192 \times 144$ pixels. 
Table 4. Runtime comparison. Compared to R-PCA, FR-PCA speeds-up the computation by a factor of 18 .

\begin{tabular}{|l|c|c|c|c|c|c|} 
& \multicolumn{6}{|c|}{ Mean Runtime [s] } \\
\hline Occlusion & $0 \%$ & $10 \%$ & $20 \%$ & $30 \%$ & $50 \%$ & $70 \%$ \\
\hline PCA & 0.006 & 0.007 & 0.007 & 0.007 & 0.008 & 0.009 \\
\hline R-PCA & 6.333 & 6.172 & 5.435 & 4.945 & 3.193 & 2.580 \\
\hline FR-PCA & 0.429 & 0.338 & 0.329 & 0.334 & 0.297 & 0.307 \\
\hline
\end{tabular}

\section{Conclusion}

We developed a novel fast robust PCA (FR-PCA) method based on an efficient two-stage outlier detection procedure. The main idea is to estimate a large number of small PCA sub-subspaces from a subset of points in parallel. Thus, for a given test sample, those sub-subspaces with the largest errors are discarded first, which reduce the number of outliers in the input data (gross outlier detection). This set - almost containing inliers - is then used to robustly reconstruct the sample by minimizing the least square reconstruction error (refinement). Since the gross outlier detection is computationally much cheaper than the refinement, the proposed method drastically decreases the computational effort for the robust reconstruction. In the experiments, we show that our new fast robust PCA approach outperforms existing methods in terms of speed and accuracy. Thus, our algorithm is applicable in practice and can be applied for real-time applications such as robust Active Appearance Model (AAM) fitting [22. Since our approach is quite general, FR-PCA is not restricted to robust image reconstruction.

\section{Acknowledgments}

This work has been funded by the Biometrics Center of Siemens IT Solutions and Services, Siemens Austria. In addition, this work was supported by the FFG project AUTOVISTA (813395) under the FIT-IT programme, and the Austrian Joint Research Project Cognitive Vision under projects S9103-N04 and S9104N04.

\section{References}

1. Jolliffe, I.T.: Principal Component Analysis. Springer, Heidelberg (2002)

2. Murase, H., Nayar, S.K.: Visual learning and recognition of 3-d objects from appearance. Intern. Journal of Computer Vision 14(1), 5-24 (1995)

3. Kirby, M., Sirovich, L.: Application of the karhunen-loeve procedure for the characterization of human faces. IEEE Trans. on Pattern Analysis and Machine Intelligence 12(1), 103-108 (1990)

4. Turk, M., Pentland, A.: Eigenfaces for recognition. Journal of Cognitive Neuroscience 3(1), 71-86 (1991)

5. Wang, Y., Huang, K., Tan, T.: Human activity recognition based on $\mathrm{r}$ transform. In: Proc. CVPR (2008) 
6. Tai, Y.W., Brown, M.S., Tang, C.K.: Robust estimation of texture flow via dense feature sampling. In: Proc. CVPR (2007)

7. Lee, S.M., Abbott, A.L., Araman, P.A.: Dimensionality reduction and clustering on statistical manifolds. In: Proc. CVPR (2007)

8. Cootes, T.F., Taylor, C.J., Cooper, D.H., Graham, J.: Active shape models - their training and application. Computer Vision and Image Understanding 61, 38-59 (1995)

9. Cootes, T.F., Edwards, G.J., Taylor, C.J.: Active appearance models. IEEE Trans. on Pattern Analysis and Machine Intelligence 23(6), 681-685 (2001)

10. Huber, P.J.: Robust Statistics. John Wiley \& Sons, Chichester (2004)

11. Hampel, F.R., Ronchetti, E.M., Rousseeuw, P.J., Stahel, W.A.: Robust Statistics: The Approach Based on Influence Functions. John Wiley \& Sons, Chichester (1986)

12. $\mathrm{Xu}, \mathrm{L} .$, Yuille, A.L.: Robust principal component analysis by self-organizing rules based on statistical physics approach. IEEE Trans. on Neural Networks 6(1), 131143 (1995)

13. Torre, F.d., Black, M.J.: A framework for robust subspace learning. Intern. Journal of Computer Vision 54(1), 117-142 (2003)

14. Roweis, S.: EM algorithms for PCA and SPCA. In: Advances in Neural Information Processing Systems, pp. 626-632 (1997)

15. Tipping, M.E., Bishop, C.M.: Probabilistic principal component analysis. Journal of the Royal Statistical Society B 61, 611-622 (1999)

16. Skočaj, D., Bischof, H., Leonardis, A.: A robust PCA algorithm for building representations from panoramic images. In: Heyden, A., Sparr, G., Nielsen, M., Johansen, P. (eds.) ECCV 2002. LNCS, vol. 2353, pp. 761-775. Springer, Heidelberg (2002)

17. Rao, R.: Dynamic appearance-based recognition. In: Proc. CVPR, pp. 540-546 (1997)

18. Black, M.J., Jepson, A.D.: Eigentracking: Robust matching and tracking of articulated objects using a view-based representation. In: Proc. European Conf. on Computer Vision, pp. 329-342 (1996)

19. Leonardis, A., Bischof, H.: Robust recognition using eigenimages. Computer Vision and Image Understanding 78(1), 99-118 (2000)

20. Edwards, J.L., Murase, J.: Coarse-to-fine adaptive masks for appearance matching of occluded scenes. Machine Vision and Applications 10(5-6), 232-242 (1998)

21. Geusebroek, J.M., Burghouts, G.J., Smeulders, A.W.M.: The Amsterdam Library of Object Images. International Journal of Computer Vision 61(1), 103-112 (2005)

22. Storer, M., Roth, P.M., Urschler, M., Bischof, H., Birchbauer, J.A.: Active appearance model fitting under occlusion using fast-robust PCA. In: Proc. International Conference on Computer Vision Theory and Applications (VISAPP), February 2009, vol. 1, pp. 130-137 (2009) 